\title{
Functional Dissection of Trigger Factor and DnaK: Interactions with Nascent Polypeptides and Thermally Denatured Proteins
}

\author{
Elke Schaffitzel, Stefan Rüdigera, Bernd Bukau* \\ and Elke Deuerling* \\ Institut für Biochemie und Molekularbiologie, \\ Universität Freiburg, Hermann-Herder-Str. 7, \\ D-79104 Freiburg, Germany \\ ${ }^{*}$ Corresponding authors
}

In Escherichia coli, the ribosome-associated Trigger Factor (TF) cooperates with the DnaK system in the folding of newly synthesized cytosolic polypeptides. Here we investigated the functional relationship of TF and DnaK by comparing various functional properties of both chaperones. First, we analyzed the ability of TF and DnaK to associate with nascent polypeptides and full-length proteins released from the ribosome. Toward this end, we established an E. coli based transcription/translation system containing physiological ratios of TF, DnaK and ribosomes. In this system, TF can be crosslinked to nascent polypeptides of $\sigma^{32}$. No TF crosslink was found to full-length $\sigma^{32}$, which is known to be a DnaK substrate. In contrast, DnaK crosslinked to both nascent and full-length $\sigma^{32}$. DnaK crosslinks critically depended on the type of chemical crosslinker. Crosslinks represent specific substratechaperone interactions since they relied on the association of the nascent polypeptides with the substrate binding pocket of DnaK. While DnaK is known to be the major chaperone to prevent protein aggregation under heat shock conditions, we found that TF did not prevent aggregation of thermally unfolded proteins in vitro and was not able to complement the heat-sensitive phenotype of a $\Delta d n a K 52$ mutant in vivo. These data indicate that TF and DnaK show strong differences in their ability to prevent aggregation of denatured proteins and to associate with native like substrates, but share the ability to associate with nascent polypeptides.

Key words: Hsp70/Prevention of aggregation/Protein folding/Protein synthesis/ $\sigma^{32}$.

\section{Introduction}

In Escherichia coli, Trigger Factor (TF) as well as the DnaK and the GroEL chaperone systems assist the folding of newly synthesized proteins. TF associates in an apparent $1: 1$ stoichiometry with ribosomes and displays chaperone and peptidyl-prolyl-cis/trans isomerase (PPlase) activities in vitro (Stoller et al., 1995; Hesterkamp et al., 1996; Scholz et al., 1997). TF interacts with virtually all nascent chains, even as short as 57 aa, and is located on the large ribosomal subunit near the exit channel for polypeptides. It was therefore proposed that TF is the first chaperone that interacts with nascent chains and assists co-translational folding processes (Valent et al., 1995, 1997; Hesterkamp et al., 1996; Bukau et al., 2000). TF is present in a $2-3$ fold molar excess over ribosomes in the cell (Lill et al., 1988). Up to now it is not known whether TF, which is not in complex with ribosomes, has additional functions in vivo.

Beyond the interaction with the ribosome associated TF a subset of newly synthesized polypeptides requires further assistance for folding by the DnaK or/and the GroEL system (Hartl, 1996; Bukau et al., 2000). GroEL associates with approximately $10-15 \%$ of newly synthesized polypeptides predominantly in a post-translational manner (Fayet et al., 1989; Horwich et al., 1993; Ewalt et al., 1997). DnaK interacts with approximately $9-18 \%$ of newly synthesized proteins including polypeptides in statu nascendi, indicating that the DnaK system may act co- and post-translationally (Deuerling et al., 1999; Teter et al., 1999).

Deletion of the dnaK gene leads to a cold- and heatsensitive phenotype, but the cells grow between $30^{\circ} \mathrm{C}$ and $37^{\circ} \mathrm{C}$ and have no severe folding defects at the permissive temperature (Bukau and Walker, 1989a; Hesterkamp and Bukau, 1998). Cells with a deletion of the tig gene show no defects and are viable between $15^{\circ}$ and $42^{\circ} \mathrm{C}$. However, deletion of both genes, dnaK and tig, causes synthetic lethality at all temperatures tested (Deuerling et al., 1999; Teter et al., 1999) and goes along with extensive aggregation of more than 300 cytosolic proteins (Deuerling et al., 1999; Deuerling and Bukau, unpublished results). E. coli thus tolerates the loss of either DnaK or TF in the presence of the other chaperone, suggesting complementary functions of the two chaperones. This is further substantiated by the finding that in cells lacking TF a 2-3 fold higher amount of newly synthesized proteins associate with DnaK (Deuerling et al., 1999; Teter et al., 1999).

In this study we dissected the functional properties of TF and DnaK. In order to study association with nascent 
polypeptides, we developed a cell-free transcription/ translation system with physiological molar ratios of ribosomes, TF, DnaK and its co-chaperones. Furthermore, we analyzed to which extent TF can back up the DnaK system with regard to DnaK's function as a modulator of the heat shock response and as the major chaperone in preventing aggregation of heat denatured proteins. We found that DnaK is a highly versatile chaperone, which associated with protein substrates in nascent, unfolded and native states. In contrast, TF is functionally restricted to nascent substrates. This chaperone is inefficient in prevention of aggregation of thermally unfolded cellular proteins and in association with full-length $\sigma^{32}$, the transcription activator of the heat shock response and natural substrate of DnaK in its native state.

\section{Results}

\section{Elaboration of a Transcription/Translation System with Defined Chaperone Content}

To investigate the functional overlap between TF and the DnaK system we analyzed their association with substrates in the nascent state and in the native state after release from the ribosome. For that purpose we developed a cell-free transcription/translation system (t/t-system) derived from the $E$. coli strain MC4100, a wild-type (wt) strain with respect to TF and DnaK. The t/t-system was prepared by the separation of the soluble lysate (S150 extract) from ribosomes and subsequent fractionation of the S150 lysate. The method used (Behrmann et al., 1998) provided a concentrated and efficient $t / t-$ system that is free of P48 (Ffh), SecA and SecB activities important for protein secretion (Beck et al., 2000). However, this system was not further characterized for its

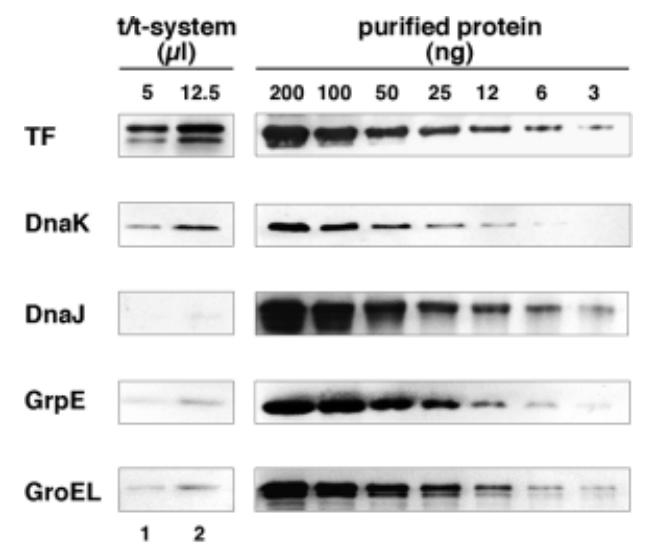

Fig. 1 Levels of Cytosolic E. coli Chaperones in the t/t-System. The in vitro t/t-system contained $6 \mu$ l translation extract, $100 \mathrm{nM}$ ribosomes and 10 units $\mathrm{T} 7$ polymerase per $25 \mu$ synthesis reaction. Five $\mu \mathrm{l}$ and $12.5 \mu \mathrm{l}$ aliquots of this mixture were subjected to immunoblot analysis using chaperone-specific antisera (lane 1 and 2) except for GroEL where 12.5 and $25 \mu$ l were applied. Serial dilutions of purified proteins served as a standard and allowed quantification of the signals in the linear range. content of TF, the components of the DnaK system (DnaK, co-chaperones DnaJ and GrpE) and the GroEL system (GroEL and co-chaperone GroES). In E. coli the physiological ratios of TF:DnaK:GroEL in their active oligomeric states are approximately 3:2:0.2:1 compared to ribosomes (Lill et al., 1988; Hesterkamp and Bukau, 1998; Mogk et al., 1999; Teter et al., 1999). We determined the chaperone content of our $\mathrm{t} / \mathrm{t}$-system by quantitative immunoblotting (Figure 1) and found that all chaperones except GrpE were underrepresented compared to the ribosome concentration (100 nM) used in this system. Therefore we added purified chaperones to obtain final concentrations of $300 \mathrm{~nm}$ TF, $200 \mathrm{~nm}$ DnaK and $20 \mathrm{~nm}$ DnaJ, which represents physiologically relevant relative molar ratios of these chaperones. We did not supplement the GroEL system since this chaperone is supposed to act exclusively post-translationally (Ewalt et al., 1997).

\section{Crosslinking of DnaK to Nascent Polypeptides Depends on the Chemical Crosslinker}

It has been reported that arrested nascent chains of proOmpA efficiently crosslink to TF in a t/t-system as

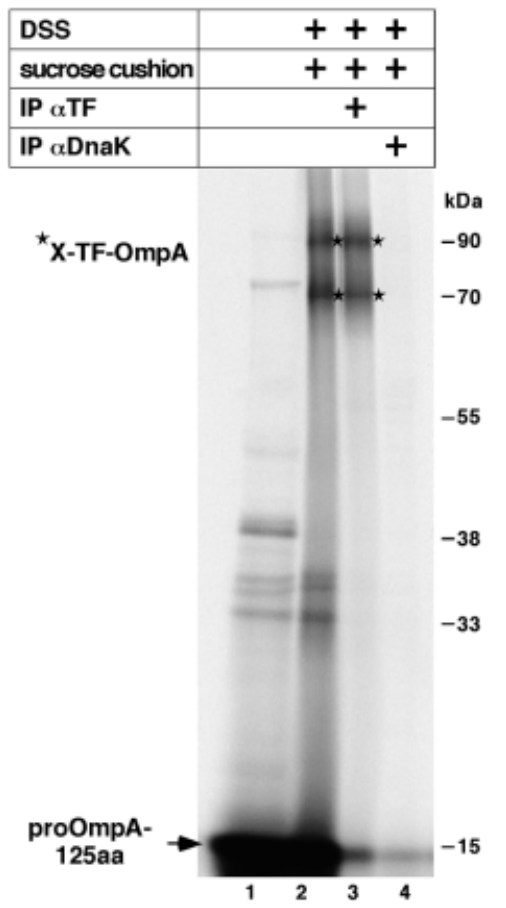

Fig. 2 Trigger Factor But Not DnaK Can Be Crosslinked to Nascent proOmpA by DSS.

Arrested nascent proOmpA polypeptides of 125 aa length were synthesized in the $t / t-s y s t e m$ in the presence of physiological chaperone ratios (lane 1; please note that additional minor bands visible in the autoradiography correspond to either residual full-length proOmpA or endogenous background products of the $\mathrm{t} / \mathrm{t}$-system). After crosslinking with DSS RNCs were purified by centrifugation through a sucrose cushion (lane 2). Aliquots were immunoprecipitated using antisera against TF (lane 3 ) and DnaK (lane 4). All samples were analyzed by SDS-PAGE; [ ${ }^{35}$ S]methionine labeled proteins were detected by phosphorimaging. The asterisks indicate crosslinking of TF. 
prepared here using the chemical crosslinker DSS (disuccinimidyl suberate) (Beck et al., 2000). We generated $\left[{ }^{35} \mathrm{~S}\right]-$ methionine labeled arrested nascent proOmpA polypeptides of 125 aa length as a model system to establish the optimal crosslinking not only for TF but also for DnaK. To create nascent chains of defined lengths, we used an antisense oligonucleotide approach, in which a DNA oligonucleotide hybridizes with the mRNA and the hybrid region is subsequently hydrolyzed by $\mathrm{RNaseH}$, resulting in truncated mRNA (Behrmann et al., 1998). Ribosome/nascent chain complexes (RNCs) were crosslinked to associated chaperones using DSS. Two crosslinking products of approximately 70 and $90 \mathrm{kDa}$ were obtained (Figure 2), both of which were identified as complexes between nascent proOmpA and TF. These two TF crosslink adducts were already found earlier using this $t / t-s y s-$ tem (Beck et al. , 2000). The $70 \mathrm{kDa}$ complex corresponds arithmetically to the complex of TF and proOmpA, whereas the $90 \mathrm{kDa}$ complex migrates with an aberrant mobility for unknown reasons. In contrast to earlier studies (Behrmann et al., 1998; Beck et al., 2000), our t/t-system was supplemented with the DnaK system to establish physiological ratios of chaperones, ribosomes and nascent polypeptides. We nevertheless failed to detect any crosslinking between DnaK and nascent proOmpA. The used DSS crosslinker is a homo-bifunctional NHS-ester which crosslinks primary amines not more than $11.4 \AA$ apart from each other. Targets are preferentially $\mathrm{N}$-termini of polypeptides and $\varepsilon$-amines of lysyl residues. To test whether DSS is suitable to generate crosslinks between nascent proOmpA polypeptides and DnaK, we localized the lysyl residues on the surface of the DnaK substrate binding domain structure (Zhu et al., 1996). In Figure 3 lysyl residues surrounding the actual substrate binding pocket are indicated by numbers. The nearest lysyl residue (residues 446 of the DnaK sequence) that may be crosslinked to a substrate bound in the substrate binding pocket is approximately $10 \AA$ away from the binding cavity. This distance is at the borderline for DSS crosslinking. Considering that the partner lysyl residue of the bound substrate is perhaps not in optimal distance, this may lead to inefficient or no crosslinking using DSS, although the chaperone-substrate complex is actually formed.

Therefore, we next tested the hetero-bifunctional crosslinker EDC [1-ethyl-3-(3-dimethylaminopropyl)-carbodiimide]. EDC first reacts with carboxyl groups thereby forming an amine reactive intermediate. Subsequently, amino groups are covalently linked to the carboxyl-termini of the first reaction partner without any spacing. In the substrate binding domain of DnaK five amino acids with carboxyl side chains are located adjacent to the substrate binding pocket (Glu402, Glu430, Glu431, Glu402, Asp530, Asp540) and offer additional crosslinking possibilities within a distance ranging from approximately $8 \AA$ for Glu402 and Glu430 up to about $15 \AA ̊$ for Asp530 (Figure 3). The results of crosslinking of [ $\left.{ }^{35} \mathrm{~S}\right]$-labeled arrested proOmpA nascent chains (125 aa) with EDC in the t/tsystem are shown in Figure 4. Two crosslinking products were identified, a major adduct at approximately $70 \mathrm{kDa}$ and a minor product of approximately $90 \mathrm{kDa}$. Co-im-

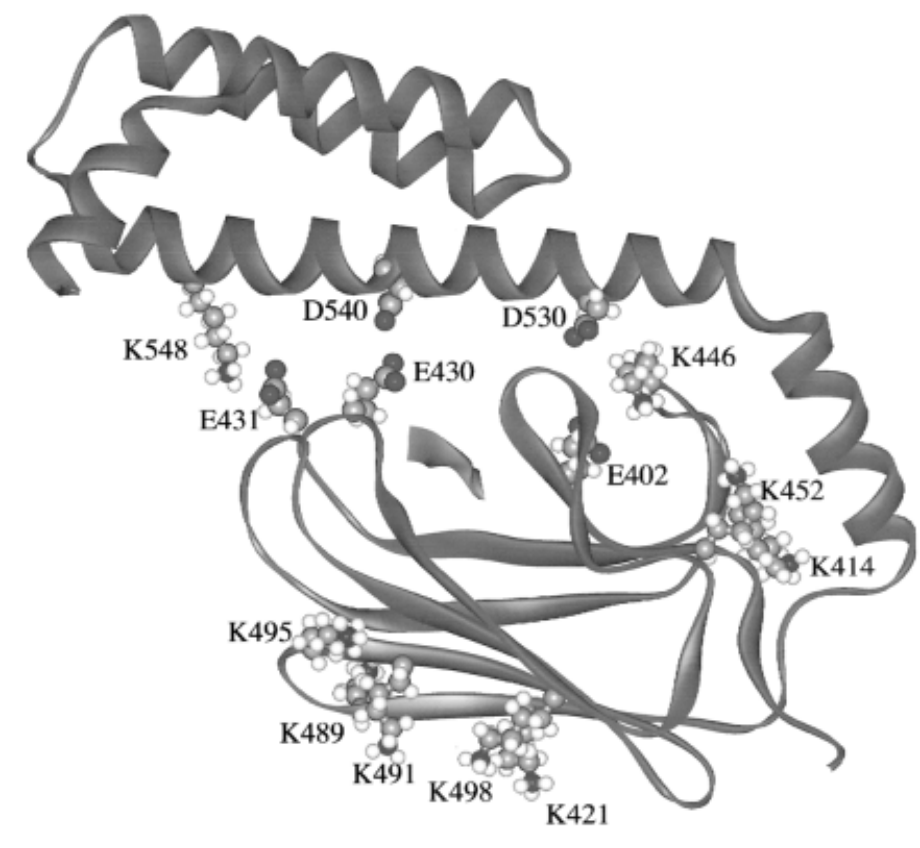

Fig. 3 Potential Amino Acid Side Chains for Crosslinking in the Substrate Binding Domain of DnaK.

Secondary structure representation of a DnaK fragment representing most of its substrate binding domain (Zhu et al., 1996). The amino acid side chains of lysyl, aspartyl and glytamyl residues that are potential targets of the chemical crosslinkers DSS (lysyl residues) and EDC (lysyl, aspartyl or glytamyl residues) are shown in space filling representation and indicated by their residue number of the fulllength DnaK. The co-crystallized bound peptide substrate is represented by the isolated fragment in the center. The representation of the DnaK substrate binding domain was created using WebLabViewer. 


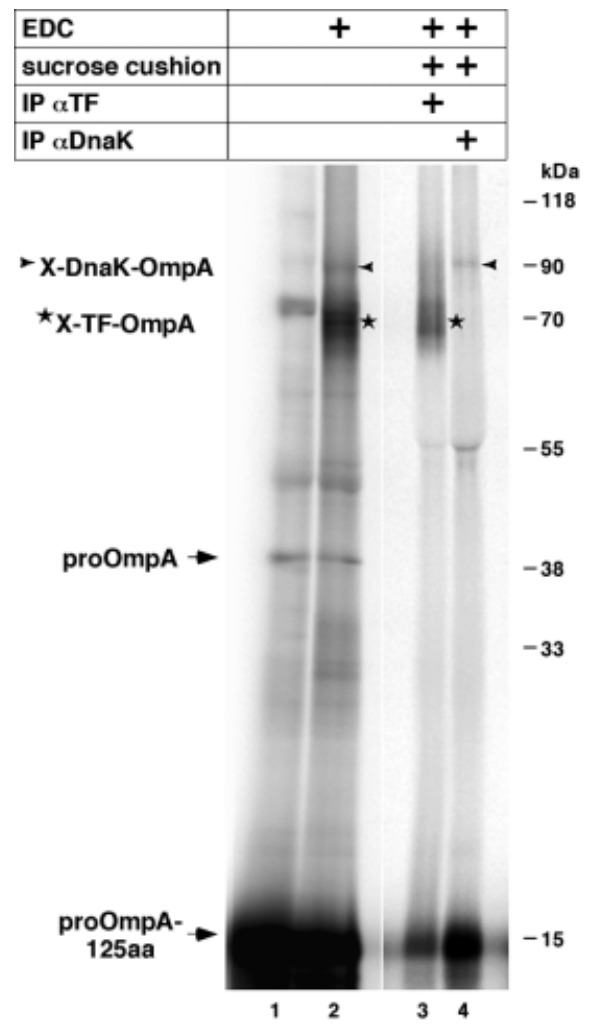

Fig. 4 EDC Crosslinking Reveals Interaction of both Trigger Factor and DnaK with Nascent proOmpA.

ProOmpA nascent chains of 125 aa length were synthesized in the $t / t-s y s t e m$ in the presence of physiological chaperone ratios (lane 1). Crosslinking with EDC (lane 2) was followed by centrifugation through a sucrose cushion and immunoprecipitation with anti-TF and anti-DnaK antibodies (lanes 3 and 4). The samples were analyzed by SDS-PAGE and phosphorimaging. Crosslinking products with DnaK (arrow) and TF (asterisk) are indicated.

munoprecipitation identified TF as the crosslinking partner of the $70 \mathrm{kDa}$ product and DnaK as partner of the 90 $\mathrm{kDa}$ product. The fuzziness of the crosslinking products is likely due to the fact that the chemical crosslinker may link each protein at various positions.

We showed that TF as well as DnaK could be crosslinked to nascent proOmpA. Furthermore, we conclude that a careful selection of the appropriate crosslinking agent is necessary to exclude wrong negative results, since crosslinking of DnaK to substrates critically depended on the specificity of the crosslinker and the accessibility of the residues for crosslinking in the chaperone and the substrate.

\section{Crosslinking of DnaK to Polypeptides Represents a Specific Chaperone-Substrate Interaction}

In order to prove that the crosslinking products obtained in our $\mathrm{t} / \mathrm{t}$-system represent real substrate-chaperone complexes, we made use of two variants of DnaK. The DnaK-V436F mutant protein is dramatically impaired in substrate interaction due to $\mathrm{F} 436$ blocking the hydrophobic substrate binding pocket (Mayer et al., 2000). The
DnaK-Q424C mutant protein can be modified with the UV-inducible crosslinker BPIA (benzophenone-4-iodoacetamide) via the single C424 residue, thereby allowing specific crosslinking between DnaK and substrates bound to the substrate binding cavity (Laufen et al., 1999). To probe the interaction of the two DnaK variants with nascent substrates, we used proOmpA nascent chains generated in our $\mathrm{t} / \mathrm{t}$-system. Since this system contains already $60 \mathrm{~nm}$ wt DnaK, we added the DnaK mutant proteins or wt DnaK as control in a 20 -fold molar excess over ribosomes to efficiently compete with the endogenous DnaK. UV exposure of the $\mathrm{t} / \mathrm{t}$-system led to crosslinking of DnaK-Q424C carrying BPIA to nascent proOmpA. Figure 5 (lanes 5,6$)$ showed two crosslinking products of about 90 and $85 \mathrm{kDa}$, respectively. Although crosslinking efficiency was much lower compared to EDC crosslinking, the $90 \mathrm{kDa} U \mathrm{~V}$-adduct corresponds to the $90 \mathrm{kDa}$ crosslink detected with EDC (Figure 5, compare lanes 2 and 3 with lanes 5 and 6). Furthermore, addition of DnaK-V436F mutant protein and subsequent EDC crosslinking led to a dramatically decreased efficiency of formation of the $90 \mathrm{kDa}$ crosslink product between DnaK and the substrate. The residual crosslink visible in Figure 5, lane 8, may result from the endogenous wild-type DnaK. Taken together, these experiments show that crosslinking with EDC specifically monitors chaperone-substrate interactions.

\section{TF Does Not Associate with Native $\sigma^{32}$, a Substrate Protein of DnaK}

$\sigma^{32}$ is the heat shock gene-specific sigma subunit of RNA polymerase and is, in its native state, a substrate of DnaK in vivo. The association with DnaK modulates the activity and half-life of $\sigma^{32}$ (Georgopoulos et al., 1994). We chose $\sigma^{32}$ as a model substrate to investigate the extent by which the functions of TF and DnaK overlap. We first analyzed the association of both chaperones with nascent $\sigma^{32}$ by generating arrested nascent chains of 215 aa length in the t/t-system. EDC crosslinking resulted in several products of sizes ranging from $70 \mathrm{kDa}$ to $120 \mathrm{kDa}$ (Figure 6A, lane 2). Co-immunoprecipitation revealed that the crosslinking products correspond either to crosslinks of nascent $\mathrm{o}^{32}$ with TF (70 and $85 \mathrm{kDa}$ adducts) or with DnaK $(75,85,100$ and $120 \mathrm{kDa})$. No crosslinking product was large enough to accommodate the simultaneous crosslinking of both chaperones to the same nascent chain, although formation of a ternary $\sigma^{32}$-DnaK-TF complex cannot be excluded. Several contact sites between nascent $\sigma^{32}$ and each chaperones appear to exist since multiple crosslinking species with diverse mobilities in the SDS-PAGE were detectable.

We then analyzed the interaction of TF and DnaK with full-length $\sigma^{32}$ in the t/t-system. After synthesis of fulllength $\sigma^{32}$ and its release from ribosomes, the folding to its native state was allowed to proceed by incubation at $37^{\circ} \mathrm{C}$ for at least $10 \mathrm{~min}$. Subsequently EDC was added to crosslink chaperones associated with native $\sigma^{32}$ (Fig- 


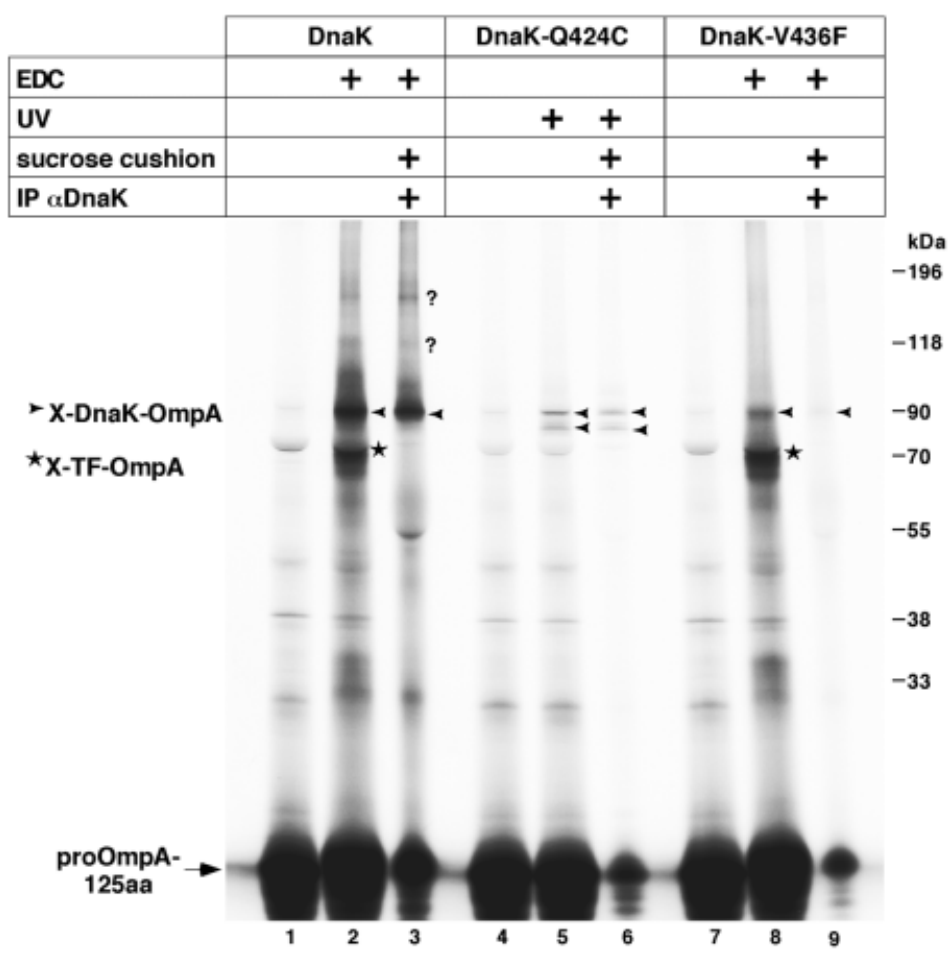

Fig. 5 DnaK Crosslinks Represent Specific Chaperone-Substrate Interactions.

Arrested nascent proOmpA polypeptides of 125 aa length were synthesized in the t/t-system in the presence of $2 \mu \mathrm{M}$ DnaK, DnaKQ424C or DnaK-V436F and $0.2 \mu \mathrm{m}$ DnaJ. For crosslinking the samples were either treated with EDC or (in the case of DnaK-Q424C) UVirradiated as indicated. After crosslinking the RNCs were purified by centrifugation through a sucrose cushion and immunoprecipitated using antisera against DnaK. All samples were analyzed by SDS-PAGE and phosphorimaging. Crosslinking products with DnaK (arrow) and TF (asterisk) are indicated. Question marks indicate DnaK crosslinks of high molecular size only present when DnaK was added in 20-fold molar excess over ribosomes. Lane 9: please note that co-immunoprecipitation efficiency of DnaK crosslink products is low most probably due to less efficient recognition of DnaK-V436F by the DnaK antibodies.

ure 6B, lanes 1 and 2). While no crosslinking to TF occurred (Figure 6B, lanes 2 and 3) crosslinking to DnaK resulted in an adduct of about $100 \mathrm{kDa}$ (Figure 6B, lanes 2 and 4). Three additional crosslinking products were found in the high molecular weight region $>120 \mathrm{kDa}$, which probably represent complexes between RNA polymerase subunits (RNAP) and $\sigma^{32}$. The addition of an excess of DnaK abolished the three high molecular weight products (Figure 6B, lane 6). Since DnaK is known to compete with RNAP for association with native $\sigma^{32}$ (Arsène et al., 1999), this further substantiates that these crosslinks correspond to the association of $\sigma^{32}$ with the RNAP in the $t / t$-system.

Taken together, these results show that DnaK associates with both, nascent and native $\sigma^{32}$. TF, however, specifically crosslinks to nascent $\sigma^{32}$ but not to the native protein, indicating that TF has no effect on the regulatory interaction of DnaK with $\sigma^{32}$.

\section{TF Cannot Substitute for DnaK in Preventing the Aggregation of Unfolded Proteins}

DnaK is known to be the most efficient chaperone in preventing aggregation of thermally unfolded proteins in $E$. coli (Mogk et al., 1999). To further analyze the extent by which TF and DnaK overlap in their chaperone activities, we compared the capacity of both chaperones to prevent aggregation of thermally denatured proteins. We prepared cell extracts from $\Delta d n a K 52$ cells grown at $30^{\circ} \mathrm{C}$. Heat treatment of this extract caused massive aggregation of proteins as evidenced by the electrophoretic separation of the aggregated pellet fraction on SDS-PAGE (Figure 7, lane 1: control with BSA added; Mogk et al., 1999). The exogenous addition of DnaK and its co-chaperones prior to the heat treatment suppressed aggregation in a concentration dependent manner (Figure 7, lanes 2 and 3). In contrast, when TF was added to the same or even higher concentration than DnaK, protein aggregation was not efficiently prevented. The concentration of aggregating proteins in this extract was approximately 5-10 $\mu \mathrm{M}$ (Mogk et al., 1999). The supplementation with 7.5 up to $15 \mu \mathrm{M}$ TF established at least a 1:1 stoichiometry of non-ribosomal TF and potential substrates, irrespective of the endogenous TF partially complexed with ribosomes. To test whether TF and DnaK act cooperatively, we supplemented the extract with DnaK and TF in various combinations (Figure 7, lanes 6-8). However, the degree of aggregation prevention depended only on the presence of the DnaK system and was not altered by further addition of TF. We conclude that, in 


\begin{tabular}{l|r|}
\hline EDC & +++ \\
\hline sucrose cushion & $+\boldsymbol{+}$ \\
\hline IP $\alpha$ TF & + \\
\hline IP $\alpha$ DnaK & + \\
\hline
\end{tabular}
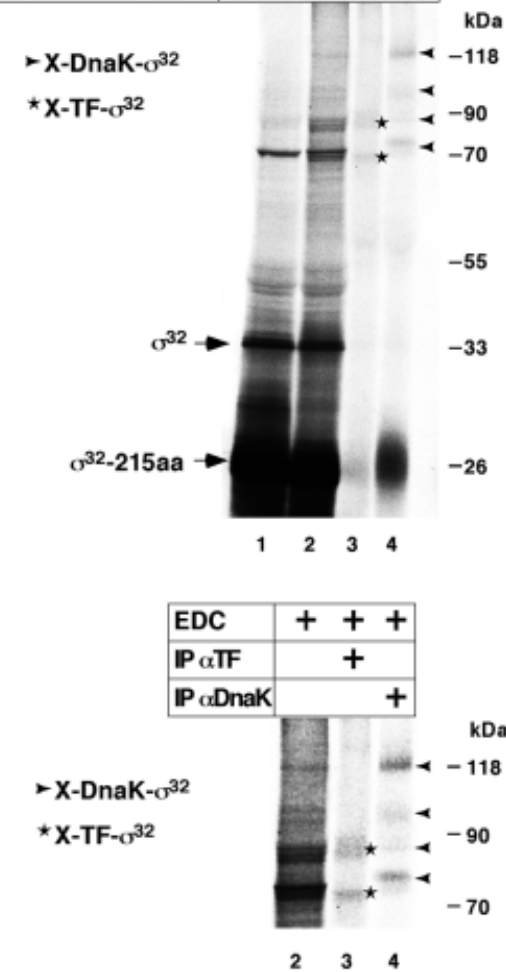

B

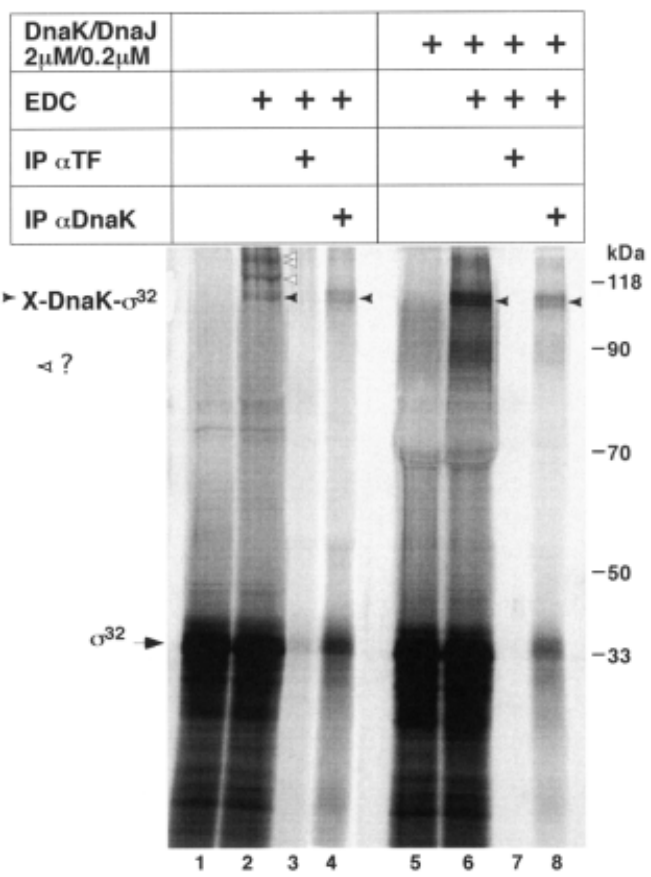

Fig. 6 Trigger Factor and DnaK Both Bind to Nascent $\sigma^{32}$ Polypeptides Whereas Only DnaK Crosslinks to Native $\sigma^{32}$.

(A) $\sigma^{32}$ arrested nascent chains of 215 aa length were synthesized in the presence of physiological ratios of chaperones (lane 1). After crosslinking with EDC (lane 2) the RNCs were purified by centrifugation through a sucrose cushion and subjected to immunoprecipitation using antisera directed against TF and DnaK (lanes 3 and 4). Crosslinking products were analyzed by SDS-PAGE. The panel at the bottom is a magnification of the lanes 2 to 4 for better visualization. (B) Full-length $\sigma^{32}$ was generated in the t/t-system under physiological chaperone ratios (lane 1) or in the presence of $2 \mu \mathrm{M}$ DnaK and $0.2 \mu \mathrm{M}$ DnaJ (20-fold molar excess over ribosomes, lane 5). After synthesis EDC was added for crosslinking (lane 2 and 6). Aliquots were immunoprecipitated using antisera against TF (lane 3 and 7 ) and DnaK (lane 4 and 8). All samples were analyzed by SDS-PAGE and phosphorimaging. The black arrow indicates the position of the crosslinking product specific for DnaK, white arrows mark potential crosslinks with RNA polymerase, and asterisks in (A) indicate TF crosslinks.

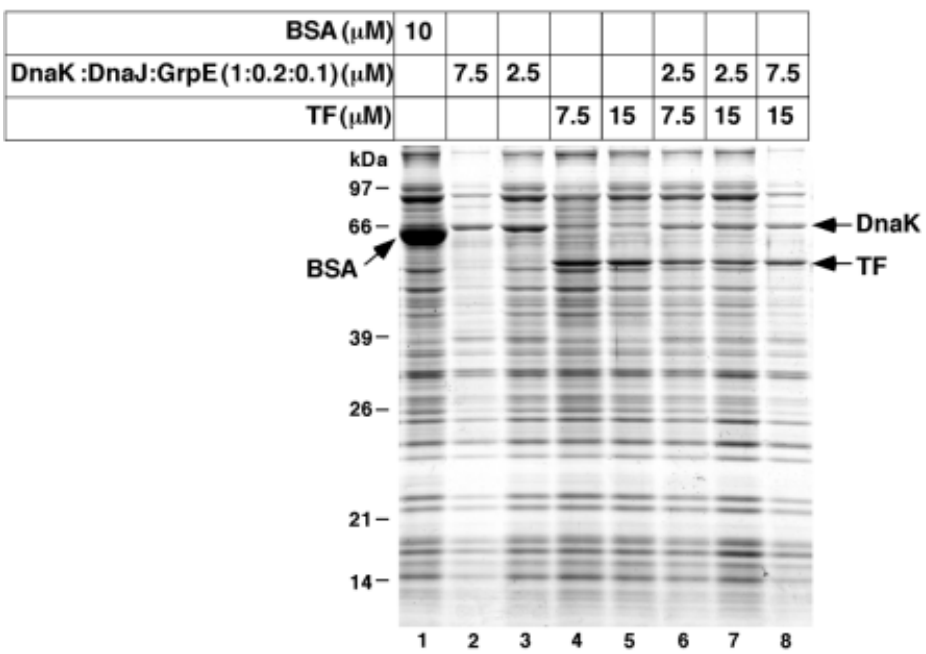

Fig. 7 TF Cannot Efficiently Prevent the Aggregation of Thermally Unfolded Proteins in Vitro.

Coomassie Blue stained SDS-PAGE of heat-denatured aggregated proteins. Extracts of total soluble cellular proteins isolated from $\triangle d n a K 52$ cells grown at $30^{\circ} \mathrm{C}$ were incubated for $15 \mathrm{~min}$ at $45^{\circ} \mathrm{C}$ in the presence of $\mathrm{BSA}$ (control) or chaperones. Aggregated material was pelleted as described (Mogket al., 1999), solubilized in 30 I SDS-sample buffer and separated by electrophoresis using a 12\% SDS-PAGE. 


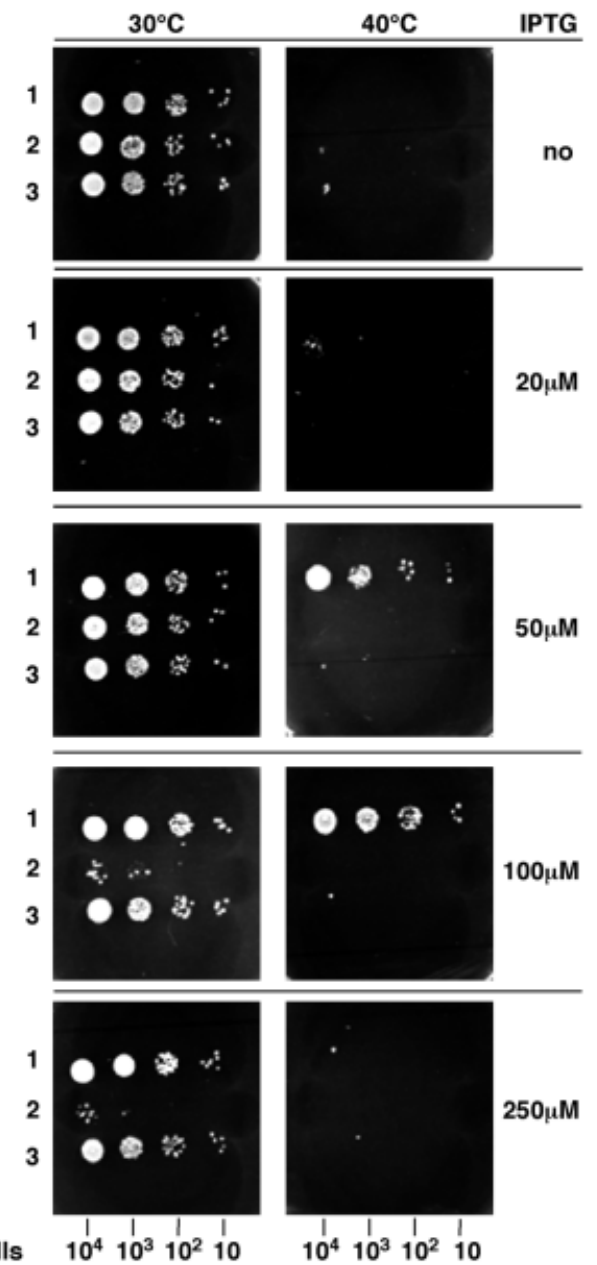

Fig. 8 Trigger Factor Is Unable to Complement the Heat-Sensitive Phenotype of $\Delta d n a K 52$ Cells.

$\Delta d n a K 52$ cells carrying a plasmid expressing lacl $^{\mathrm{Q}}$ repressor were transformed with plasmids either expressing TF (lanes 2) or DnaK (lanes 1) by IPTG induction. For a control cells were transformed with the vector plasmid (lanes 3 ). Cells were spotted in different dilutions on agar plates supplemented with 20,50, 100 and $250 \mu \mathrm{M}$ or without IPTG. Plates were incubated at 30 or 40 ${ }^{\circ} \mathrm{C}$ for $24 \mathrm{~h}$.

contrast to DnaK, TF is inefficient in suppression of aggregation of thermolabile proteins in vitro. TF neither substitutes for DnaK nor cooperates with the DnaK system in preventing the aggregation of bulk $E$. coli proteins during heat treatment.

To investigate whether this conclusion is also valid for the in vivo situation, we tested whether overexpression of TF can rescue the temperature-sensitive phenotype of a $\Delta$ dnaK52 strain (Figure 8). Cells lacking DnaK do not form colonies at $40{ }^{\circ} \mathrm{C}$ (Bukau and Walker, 1989b). Overexpression of the dnaK gene encoded by a plasmid under the control of an IPTG inducible promoter complements the heat-sensitive growth of $\Delta d n a K 52$ cells at $40{ }^{\circ} \mathrm{C}$ (Figure 8 , lanes 1). This phenotype could not be complemented by the overproduction of TF encoded by a plasmid under the control of an IPTG regulatable promoter. Moreover, the massive overproduction of TF after induc- tion of its synthesis with $100 \mu \mathrm{M}$ IPTG (corresponding to approx. 10 to 15 -fold of cellular TF levels) was lethal for $\Delta$ dnaK52 cells at $30{ }^{\circ} \mathrm{C}$ (Figure 8, lanes 2). This observation was also made with wt $E$. coli cells overproducing TF (S. Vorderwülbecke and E. Deuerling, unpublished results).

In summary, these data indicate that TF does not interact productively with thermally unfolded protein substrates to prevent their aggregation and therefore cannot substitute for DnaK during thermal stress conditions both in vitro and in vivo.

\section{Discussion}

The association of TF and DnaK with nascent polypeptides was investigated by using an in vitro $E$. coli based transcription/translation system. To work close to the physiological ratios of chaperones we determined the amount of chaperones present in our t/t-system and supplemented the system with chaperones that were underrepresented compared to their estimated cellular concentration (TF, DnaK, and its co-chaperone DnaJ). This approach created a transcription/translation system with defined amounts of ribosomes and chaperones and provided conditions comparable to the in vivo situation. To our knowledge this is the first described $t / t-s y s t e m$ operating under defined ribosome and chaperone conditions. This system will also be a very good tool for further investigations of chaperone functions $e . g$. by preparation of the $\mathrm{t} / \mathrm{t}$-system from chaperone mutant strains.

Using the chemical lysyl-specific crosslinker DSS we found exclusively TF covalently linked to nascent proOmpA chains whereas the bifunctional EDC crosslinker gave crosslinking adducts with TF as well as with DnaK. These results clearly indicate that crosslinking critically depends on the specificity of the used crosslinking agent and on the formed chaperone-substrate complex. Negative evidence therefore has to be interpreted with caution since it may result simply from the inability of the chemical crosslinker to covalently fix the chaperone-substrate interaction. This might be the reason that earlier studies did not detect DnaK as crosslinking partner of nascent polypeptide chains (Valent et al., 1995, 1997; Beck et al., 2000).

We investigated the functional redundancy of the ribosome-associated chaperone TF and the cytosolic DnaK chaperone system. This study indicates that TF function in vitro and in vivo is restricted on substrates in the nascent polypeptide state. This was shown by different approaches: (i) TF crosslinked to nascent $\sigma^{32}$ but an association with released full-length $\sigma^{32}$ could not be detected. In contrast, DnaK associated with both stages of this protein. (ii) We could not detect an efficient prevention of aggregation by TF of thermally denatured $E$. coli proteins. Obviously soluble TF is not able to associate efficiently with unfolded proteins, a finding that is supported by recent results showing that soluble TF has a $K_{\mathrm{D}}$ for peptide 
substrates in the high $\mu \mathrm{M}$ range (approx. $50 \mu \mathrm{M}$, D. Brehmer and B. Bukau, unpublished results). (iii) TF cannot complement the heat-sensitive phenotype of $\Delta$ dnaK52 mutant cells.

DnaK, like TF, has the capacity to associate with nascent substrates of both, proOmpA and $\sigma^{32}$ derived nascent polypeptides. Since DnaK has this broad functional spectrum, the loss of TF can be tolerated in the presence of DnaK functioning as a back up system for TF either by acting on nascent polypeptides or by assisting the refolding of unproductively folded proteins post-translationally. Conclusions in respect to the quantitative nature of the association of DnaK with nascent polypeptides could not be drawn. Recent results indicate that TF and DnaK efficiently compete with each other for binding to nascent proOmpA due to the fact the substrate binding sites of both chaperones overlap in this substrate (E. Deuerling und Bukau, unpublished results). Most likely the localization of TF next to the polypeptide exit of the ribosomes gives TF the advantage to associate much more efficiently with nascent polypeptides compared to DnaK.

\section{Materials and Methods}

\section{Culture Conditions}

For complementation analysis $\Delta d n a K 52$ cells carrying a plasmid (spectinomycin ${ }^{\mathrm{R}}$ ) expressing $\mathrm{lacl}^{\mathrm{Q}}$ repressor were transformed with plasmids (ampicillin ${ }^{\mathrm{R}}$ ) either expressing TF (Hesterkamp et al., 1997) or DnaK (Tomoyasu et al., 1998) by IPTG induction. Cells were grown overnight at $30^{\circ} \mathrm{C}$ in LB with $50 \mu \mathrm{g} / \mathrm{ml}$ spectinomycin and $100 \mu \mathrm{g} / \mathrm{ml} \mathrm{ampicillin} \mathrm{and} \mathrm{subsequently} \mathrm{spotted} \mathrm{on}$ LB plates containing $50 \mu \mathrm{g} / \mathrm{ml}$ spectinomycin and $100 \mu \mathrm{g} / \mathrm{ml}$ ampicillin.

\section{Isolation of Cell Extracts and Preparation of Aggregates}

Cells of the Escherichia coli MC4100 $\Delta$ dnaK52 mutant strain were grown in $100 \mathrm{ml} \mathrm{LB}$ medium at $30^{\circ} \mathrm{C}$ up to exponential growth phase, immediately chilled on ice for $10 \mathrm{~min}$ and subsequently harvested by centrifugation ( $10 \mathrm{~min}, 4000 \mathrm{~g}, 4^{\circ} \mathrm{C}$ ). Pellets were resuspended and washed twice in breakage buffer (50 mм HEPES pH 7.6, $150 \mathrm{~mm} \mathrm{KCl,} 20 \mathrm{~mm} \mathrm{MgCl}_{2}, 10 \mathrm{~mm}$ DTT) and lysed in a precooled french press cell at 5.6 MPa. After centrifugation ( $30 \mathrm{~min}, 30000 \mathrm{~g}, 4^{\circ} \mathrm{C}$ ), the concentration of soluble proteins in the supernatant was determined in a Bradford assay with BSA as standard. For thermal aggregation soluble cell extracts $(4 \mathrm{mg} / \mathrm{ml})$ were pre-incubated for $5 \mathrm{~min}$ at $30^{\circ} \mathrm{C}$ in the absence or presence of chaperones. ATP $(10 \mathrm{~mm})$ was added $2 \mathrm{~min}$ prior to heat shock. Samples were shifted for $15 \mathrm{~min}$ to $45^{\circ} \mathrm{C}$ and aggregated proteins were pelleted by centrifugation for 15 min at $15000 \mathrm{~g}$ and $4{ }^{\circ} \mathrm{C}$. Pellets were washed twice with icecold breakage buffer and analyzed by electrophoresis using $12 \%$ SDS-PAGE. Proteins were visualized by Coomassie Brilliant Blue staining.

\section{In Vitro Transcription/Translation and Chemical Crosslinking}

Preparation of translation extracts from MC4100 E. coli cells and generation of arrested nascent chains were performed as described (Beck et al., 2000). Extracts were analyzed for chaper- one contents by quantitative immunoblotting with specific antisera as described (Mogk et al., 1999). To obtain concentrations close to physiological molar ratios, purified TF ( $300 \mathrm{~nm}$, final concentration), DnaK (200 nM, final conc.) and DnaJ (20 nM, final conc.) were added to $100 \mathrm{~nm}$ of ribosomes in the translation extracts. For the generation of $\sigma^{32}$ the plasmid pFN476 (Sankar et al., 1993) and for proOmpA the p7170mpA plasmid (Beck et al., 2000) was used. For transcription $10 \mathrm{ng} / \mathrm{\mu l}$ of plasmid and 0.4 $\mathrm{U} / \mu \mathrm{l} \mathrm{T7}$ polymerase were added to the $\mathrm{t} / \mathrm{t}$-system (final volume of $25 \mu \mathrm{l})$ and reactions were started by supplementation with 0.3 $\mu \mathrm{Ci} / \mu \mathrm{l}\left[{ }^{35} \mathrm{~S}\right]-$ methionine. Arrested nascent chains were produced by the addition of $0.16 \mu \mathrm{g} / \mu \mathrm{l}$ antisense-oligonucleotide (5'-CGGTTTGCCGCCTGCTCTTCCCAGC-3' for $\sigma^{32}$ and 5'-TAAACGTTGGATTTAGTGTC-3' for proOmpA) together with $0.12 \mu \mathrm{g} / \mu \mathrm{l}$ anti-ssrA oligonucleotide (5'-TTAAGCTGCTAAAGCGTAGTTTCGTCGTTTGCGACTA-3). After 30 min, the crosslinker DSS (in DMSO, $2.5 \mathrm{~mm}$ final concentration) or EDC (in $\mathrm{H}_{2} \mathrm{O}, 80 \mathrm{~mm}$ final concentration) were added for $30 \mathrm{~min}$ at $30^{\circ} \mathrm{C}$, the reaction was quenched with $50 \mathrm{~mm}$ Tris/ $\mathrm{HCl} \mathrm{pH7.5}$ or $100 \mathrm{~mm}$ glycine, $10 \mathrm{~mm}$ $\mathrm{NaHCO}_{3}$, respectively, for 15 min on ice and ribosomal complexes were purified as described (Hesterkamp et al., 1997). The pellet was resolubilized in PBS buffer and co-immunoprecipitation performed with DnaK- or TF-specific antisera as described (Deuerling et al., 1999). Co-immunoprecipitated material was solubilized in $25 \mu \mathrm{l} \mathrm{SDS}$-sample buffer for $6 \mathrm{~min}$ at $100{ }^{\circ} \mathrm{C}$. For control of crosslinking products one sample of the $\mathrm{t} / \mathrm{t}$-extract was precipitated with 5\% TCA immediately after crosslinking and the pellet was resolubilized in $20 \mu \mathrm{l}$ sample buffer and incubated at $100{ }^{\circ} \mathrm{C}$ for $5 \mathrm{~min}$. Samples were loaded on an SDSPAGE and crosslinking products subsequently visualized by autoradiography.

\section{UV-Crosslinking Experiments}

For crosslinking of DnaK-Q424C to nascent $\sigma^{32}$ the cysteine reactive crosslinker BPIA (benzophenon-4-iodoacetamid) was attached to $\mathrm{C} 424$ of DnaK according the protocol of Laufen et al. (1999).

After generation of arrested $\sigma^{32}$ nascent polypeptides the $t / t$ system was exposed to UV light $(365 \mathrm{~nm}, 100 \mathrm{~W}, 5 \mathrm{~cm}$ distance between the light source and the sample) for $5 \mathrm{~min}$. After sucrose cushion centrifugation the DnaK-Q424C crosslinking products were co-immunoprecipitated as described (Deuerling et al., 1999) and analyzed as described above.

\section{Acknowledgements}

We thank M.P. Mayer, G. Kramer, H. Patzelt and S. Vorderwülbecke for comments on the manuscript and discussions; $M$. Müller and C. Beck for their help establishing the t/t-system. We thank H. Patzelt for preparation of Figure 3. This work was supported by grants of the DFG (SFB388, Graduiertenkolleg) to B.B. and E.D. and the Fonds der Chemischen Industrie to B.B.

\section{References}

Arsène, F., Tomoyasu, T., Mogk, A., Schirra, C., Schulze-Specking, A., and Bukau, B. (1999). Role of region C in regulation of the heat shock gene-specific sigma factor of Escherichia coli, $\sigma^{32}$. J. Bacteriol. 181, 3552-3561.

Beck, K., Wu, L.-F., Brunner, J., and Müller, M. (2000). Discrimination between SRP- and SecA/SecB-dependent substrates involves selective recognition of nascent chains by SRP and trigger factor. EMBO J. 19, 134-143. 
Behrmann, M., Koch, H.-G., Hengelage, T., Wieseler, B., Hoffschulte, H.K., and Müller, M. (1998). Requirements for the translocation of elongation-arrested, ribosome-associated OmpA across the plasma membrane of Escherichia coli. J. Biol. Chem. 273, 13898-13904.

Bukau, B., and Walker, G.C. (1989a). Cellular defects caused by deletion of the Escherichia coli dnaK gene indicates roles for heat shock protein in normal metabolism. J. Bacteriol. 171, $2337-2346$.

Bukau, B., and Walker, G.C. (1989b). $\Delta d n a K$ mutants of Escherichia coli have defects in chromosomal segregation and pasmid maintenance at normal growth temperatures. J. Bacteriol. 171, 6030-6038.

Bukau, B., Deuerling, E., Pfund, C., and Craig, E.A. (2000). Getting newly synthesized proteins into shape. Cell 101, 119-122.

Deuerling, E., Schulze-Specking, A., Tomoyasu, T., Mogk, A., and Bukau, B. (1999). Trigger factor and DnaK cooperate in folding of newly synthesized proteins. Nature 400, 693-696.

Ewalt, K.L., Hendrick, J.P., Houry, W.A., and Hartl, F.U. (1997). In vivo observation of polypeptide flux through the bacterial chaperonin system. Cell 90, 491-500.

Fayet, O., Ziegelhoffer, T., and Georgopoulos, C. (1989). The groES and groEL heat shock gene products of Escherichia coli are essential for bacterial growth at all temperatures. J. Bacteriol. 171, 1379-1385.

Georgopoulos, C., Liberek, K., Zylicz, M. and Ang, D. (1994). Properties of the heat shock proteins of Escherichia coli and the autoregulation of the heat shock response. In: The biology of heat shock proteins and molecular chaperones, R.I. Morimoto, A. Tissières and C. Georgopoulos, eds. (Cold Spring Harbor, NY, USA: Cold Spring Harbor Laboratory Press), pp. $209-250$.

Hartl, F.U. (1996). Molecular chaperones in cellular protein folding. Nature 381, 571-580.

Hesterkamp, T., and Bukau, B. (1998). Role of the DnaK and HscA homologs of Hsp70 chaperones in protein folding in $E$. coli. EMBO J. 17, 4818-4828.

Hesterkamp, T., Hauser, S., Lütcke, M., and Bukau, B. (1996). Escherichia coli trigger factor is a prolyl-isomerase that associates with nascent polypeptide chains. Proc. Natl. Acad. Sci. USA 93, 4437-4441.

Hesterkamp, T., Deuerling, E., and Bukau, B. (1997). The aminoterminal 118 amino acids of Escherichia coli trigger factor constitute a domain that is necessary and sufficient for binding to ribosomes. J. Biol. Chem. 272, 21865-21871.

Horwich, A.L., Brooks Low, K., Fenton, W.A., Hirshfield, I.N., and Furtak, K. (1993). Folding in vivo of bacterial cytoplasmic proteins: role of GroEL. Cell 74, 909-917.

Laufen, T., Mayer, M.P., Beisel, C., Klostermeier, D., Reinstein, J., and Bukau, B. (1999). Mechanism of regulation of Hsp70 chaperones by DnaJ co-chaperones. Proc. Natl. Acad. Sci. USA 96, 5452-5457.

Lill, R., Crooke, E., Guthrie, B., and Wickner, W. (1988). The 'trigger factor cycle' includes ribosomes, presecretory proteins and the plasma membrane. Cell 54, 1013-1018.

Mayer, M.P., Schröder, H., Rüdiger, S., Paal, K., Laufen, T., and Bukau, B. (2000). Multistep mechanism of substrate binding determines chaperone activity of Hsp70. Nature Struct. Biol. 7, 586-593.

Mogk, A., Tomoyasu, T., Goloubinoff, P., Rüdiger, S., Röder, D., Langen, H., and Bukau, B. (1999). Identification of thermolabile $E$. coli proteins: prevention and reversion of aggregation by DnaK and ClpB. EMBO J. 18, 6934-6949.

Sankar, P., Hutton, M.E., VanBogelen, R.A., Clark, R.L., and Neidhardt, F.C. (1993). Expression analysis of cloned chromosomal segments of Escherichia coli. J. Bacteriol. 175, 51455152.

Scholz, C., Stoller, G., Zarnt, T., Fischer, G., and Schmid, F.X. (1997). Cooperation of enzymatic and chaperone functions of trigger factor in the catalysis of protein folding. EMBO J. 16, $54-58$.

Stoller, G., Rücknagel, K.P., Nierhaus, K.H., Schmid, F.X., Fischer, G., and Rahfeld, J.-U. (1995). A ribosome-associated peptidyl-prolyl cis/trans isomerase identified as the trigger factor. EMBO J. 14, 4939- 4948.

Teter, S.A., Houry, W.A., Ang, D., Tradler, T., Rockabrand, D., Fischer, G., Blum, P., Georgopoulos, C., and Hartl, F.U. (1999). Polypeptide flux through bacterial Hsp70: DnaK cooperates with trigger factor in chaperoning nascent chains. Cell 97, $755-765$.

Tomoyasu, T., Ogura, T., Tatsuta, T., and Bukau, B. (1998). Levels of DnaK and DnaJ provide tight control of heat shock gene expression and protein repair in E. coli. Mol. Microbiol. 30, 567-581.

Valent, Q.A., Kendall, D.A., High, S., Kusters, R., Oudega, B., and Luirink, J. (1995). Early events in preprotein recognition in E. coli: interaction of SRP and trigger factor with nascent polypeptides. EMBO J. 14, 5494-5505.

Valent, Q.A., de Gier, J.-W.L., von Heijne, G., Kendall, D.A., ten Hagen-Jongman, C.M., Oudega, B., and Luirink, J. (1997). Nascent membrane and presecretory proteins synthesized in Escherichia coli associate with signal recognition particle and trigger factor. Mol. Microbiol. 25, 53-64.

Zhu, X., Zhao, X., Burkholder, W.F., Gragerov, A., Ogata, C.M., Gottesman, M., and Hendrickson, W.A. (1996). Structural analysis of substrate binding by the molecular chaperone DnaK. Science 272, 1606-1614. 